\title{
LncRNA HOXA-AS2 Facilitates Tumorigenesis and Progression of Papillary Thyroid Cancer by Modulating the miR-15a-5p/HOXA3 Axis
}

\author{
Liangfeng Jiang ${ }^{1, \dagger}$ Zhiming $\mathrm{Wu}_{1}{ }^{2, *, \dagger}$ Xingcheng Meng, ${ }^{2}$ Xiufeng $\mathrm{Chu},{ }^{2}$ \\ Hongjun Huang, and Chaoyang $\mathrm{Xu}^{3}$ \\ ${ }^{1}$ Pharmacy Department, the First People's Hospital of Fuyang Hangzhou, Hangzhou, P.R. China; ${ }^{2}$ General Surgery Department, Shaoxing Hospital of China Medical \\ University, Shaoxing, P.R. China; and ${ }^{3}$ Department of Thyroid Breast Surgery, the People's Hospital of Shaoxing, Shaoxing, P.R. China. \\ ${ }^{\dagger}$ Co-first authors.
}

The long non-coding RNA HOXA-AS2 has been found to be an oncogene in several types of human malignant tumors. However, its role in regulating the occurrence and development of papillary thyroid cancer (PTC) is still unclear. The present study investigated the function and mechanism(s) of HOXA-AS2 in PTC progression. Using quantitative real-time polymerase chain reaction, HOXA-AS2 was found to be differentially expressed in PTC tissues and cell lines. Kaplan-Meier analysis indicated that the overall survival rate of patients with higher levels of HOXA-AS2 was lower than those with relatively lower levels. Loss-of-function assays revealed that HOXA-AS2 knockdown inhibited PTC progression by inhibiting cellular proliferation, migration, and invasion and accelerating apoptosis. Mechanistically, lossof-function assays showed a positive correlation between HOXA3 and HOXA-AS2 expression. Subcellular fractionation assay results revealed abundant HOXA-AS2 expression in the cytoplasm of PTC cells. Additionally, FOXD2-AS1 was found to upregulate HOXA3 expression by binding to miR-15a-5p. Finally, rescue assays demonstrated the overall function of the HOXA-AS2/miR-15a-5p/HOXA3 axis in PTC progression. These findings will significantly contribute to further research and the development of more efficient treatments for thyroid cancer in the future.

Keywords: HOXA-AS2, miR-15a-5p, HOXA3, Wnt/ $\beta$-catenin signaling pathway, papillary thyroid cancer

\section{INTRODUCTION}

THYROID CANCER IS A highly prevalent cancer resulting from complex endocrine dyscrasia that has a high occurrence and mortality. ${ }^{1-3}$ The increased incidence of thyroid cancer over the years has threatened human health. ${ }^{4,5}$ Although some popular treatments, such as radiation, chemotherapy, and surgery, have been widely applied to treat thyroid cancer, the prognosis remains unfavorable. ${ }^{6,7}$ Therefore, development of novel therapeutic methods for this cancer is urgently needed. Thyroid cancer is commonly divided into four subtypes: papillary thyroid carcinoma (PTC; most common), anaplastic thyroid carcinoma, follicular carcinoma, and medullary thyroid carcinoma. ${ }^{8}$ The present study focused on molecular mechanisms contributing to the progression of PTC.
Long non-coding RNAs (lncRNAs) are $>200 \mathrm{nt}$ in length and do not encode proteins. ${ }^{9}$ LncRNAs are crucial modulators in multiple biological processes, such as cellular apoptosis, invasion, and migration, ${ }^{10-12}$ and their ectopic expression can promote carcinogenesis. ${ }^{13-17}$ The lncRNA HOXA-AS2 has been reported to be an oncogene in several types of human cancer. ${ }^{18-21}$ However, it is unclear whether it functions as a regulator in PTC. LncRNAs can also exert their function in human tumors by competitively sharing microRNAs (miRNAs or miRs) with mRNAs. MiRNAs are short non-coding RNAs between 19 and $22 \mathrm{nt}$ in length ${ }^{22}$ that can participate in competitive endogenous RNA (ceRNA) pathways. ${ }^{23-26}$ According to previous reports, some lncRNAs function in human malignant tumors by acting as ceRNAs. ${ }^{27-30}$ Therefore, the present

${ }^{*}$ Correspondence: Dr. Zhiming Wu, General Surgery Department, Shaoxing Hospital of China Medical University, No. 1 Huayu Road, Keqiao District, Shaoxing, 312030 P.R. China. E-mail: zhiming_wu17@163.com 
study investigated the function and mechanism(s) of HOXA-AS2 in the progression of PTC. In addition, the study also examined whether HOXA-AS2 acts as a ceRNA to regulate other nearby genes (e.g., HOXA3) by sponging miRNA, thereby modulating downstream signaling pathways (e.g., Wnt/ $\beta$-catenin).

\section{METHODS}

\section{Collection of tissue samples}

Approval from the Ethics Committee of Navy General Hospital (Beijing, P.R. China), as well as written informed consent from all included patients, was obtained prior to study onset. Matched PTC $(n=68)$ and normal $(n=68)$ tissues were obtained from PTC patients following surgical resection at Navy General Hospital. All tissue samples were directly frozen and stored in liquid nitrogen until use. PTC was confirmed in tissue samples via pathological examination. The mean expression level of HOXAAS2 in all PTC tissues was determined and used to further divide these samples into two subgroups based on high and low HOXA-AS2 expression.

\section{Cell culture}

One normal human thyroid epithelial (Nthyori3-1) and three human papillary thyroid cancer cell lines (K-1, TPC and BHP2-7) were obtained from American Type Culture Collection (Manassas, VA). All cell lines were cultured in RPMI-1640 medium supplemented with $10 \%$ fetal bovine serum (FBS, Gibco, Thermo Fisher Scientific, Inc., Waltham, MA, USA), $0.1 \mathrm{mM}$ amino acids (LGlutamine, Gibco), $1 \mathrm{mM}$ sodium pyruvate and $1 \%$ penicillin-streptomycin (Sigma-Aldrich, St. Louis, MO). Cell culture was conducted in a humidified environment with $5 \% \mathrm{CO}_{2}$ at $37^{\circ} \mathrm{C}$.

\section{Cell transfection}

MiR-15a-5p mimics/inhibitors and respective controls were purchased from GenePharma (Shanghai, P.R. China). Small interfering RNAs (siRNAs) targeted to HOXA-AS2 (si-HOXA-AS2\#1 and si-HOXAAS2\#2) or HOXA3 (si-HOXA3\#1 and si-HOXA3\#2) and control siRNA (si-NC) were also purchased from GenePharma. A pcDNA-HOXA3 plasmid was obtained from Genechem Biotech (Shanghai, P.R. China), and Lipofectamine 2000 (Invitrogen, Carlsbad, CA) was used for cellular transfections in accordance with the manufacturer's guidelines.

\section{Quantitative real-time polymerase chain reaction}

TRIzol reagent (Thermo Fisher Scientific, Waltham, MA) was used to extract total RNA from cells according to the manufacturer's instructions. The isolated RNA was used to synthesize cDNA using an All-in-One ${ }^{\mathrm{TM}}$ miRNA quantitative real-time polymerase chain reaction (qRT-PCR) detection kit (GeneCopoeia, FulenGen, P.R. China). A Prime Script $^{\text {TM }}$ RT reagent kit (Takara, Shiga, Japan) was used for reverse transcription, and qRT-PCR was repeated three times on a 96 -well PCR plate. Each well $(20 \mu \mathrm{l})$ contained $1 \mu \mathrm{L}$ of cDNA $10 \mathrm{mM}$ dNTPs and $1 \times 20 \mu \mathrm{L}$ of Platinum SYBR Green qPCR SuperMix UDG (Invitrogen). The primers sequences for HOXA-AS2 were listed as follows: GTCTGCGAAGGCCTAAAGGT (forward), GACT CCCTGATGTTCGCGTC (reverse). The primers sequences for miR-15a-5p were listed as follows: TAGCAGCACATAATGGTTTGTG (forward), CTCT ACAGCTATATTGCCAGCCA (reverse). The primers sequences for HOXA3 were listed as follows: CATCCCTTGGGTCGTCTCTC (forward), TTCTC GGGGCAAATCTGGTC (reverse). The primers sequences for U6 were listed as follows: AGGAGA CGGGAACGACAAAC (forward), GAAGAGGATT CGCTGACGGT (reverse). The primers sequences for GAPDH were listed as follows: GCTTTCTTT CCTTTCGCGCT (forward), TTTGCGGTGGAAAT GTCCTT (reverse). MiRNA and mRNA expression levels were normalized to U6-small nuclear RNA and glyceraldehyde-3-phosphate dehydrogenase (GAPDH) by the $2^{-\Delta \Delta \mathrm{Ct}}$ method. ${ }^{31}$

\section{3-(4,5-dimethylthiazol-2-yl)-2,5- diphenyltetrazolium bromide (MTT) assay}

Cells were seeded onto 96-well culture plates (Corning Costar, Corning, NY) at a density of $2 \times 10^{3}$ cells per well and cultured for $24 \mathrm{~h}$ prior to transfection. Next, $20 \mu \mathrm{L}$ of MTT solution ( $5 \mathrm{mg} /$ $\mathrm{mL}$; Sigma-Aldrich) was added and incubated in each well for $4 \mathrm{~h}$ at $37^{\circ} \mathrm{C}$. After, the media was removed from each well, and intracellular formazan crystals were dissolved by adding $200 \mu \mathrm{L}$ of dimethyl sulfoxide (Sigma-Aldrich). Cell viability was detected by measuring the absorbance at $490 \mathrm{~nm}$ on a Multiskan Ascent 354 microplate reader (Thermo Labsystems, Waltham, MA).

\section{Flow cytometry}

Following transfection, cells were collected and washed with phosphate-buffered saline before re-suspending $1 \times 10^{6}$ cells from each sample in binding buffer. Binding buffer contained phosphate buffer saline (PBS) and 1\% FBS. Then, an Annexin V-fluorescein isothiocyanate/propidium iodide kit (BD Pharmingen, San Diego, CA) was used to measure cell viability via flow cytometry (BD FACS Aria; BD Biosciences, Franklin Lakes, NJ). 


\section{Transwell ${ }^{\circledR}$ assay}

After transfection, PTC cell lines $\left(1 \times 10^{4}\right)$ were re-suspended in $200 \mu \mathrm{L}$ of serum-free medium. For both invasion and migration assays, PTC cell lines were then seeded into the upper chambers of Transwell ${ }^{\circledR}$ plates ( $8 \mu \mathrm{m}$ pore size; Corning Costar), which were coated with or without Matrigel (BD Biosciences). Medium containing 10\% FBS was used as a chemoattractant and added to the bottom chamber of each well, and cells were incubated at $37^{\circ} \mathrm{C}$ with $5 \% \mathrm{CO}_{2}$ for 24 or $48 \mathrm{~h}$ for migration and invasion assays, respectively. Afterward, cells present in the upper chamber were wiped out with cotton swabs, whereas cells that had adhered to the bottom chamber were fixed with methanol and stained with $0.1 \%$ crystal violet. Stained cells were imaged at $100 \times$ magnification under a microscope (Olympus, Tokyo, Japan).

\section{Luciferase reporter assay}

The sequences for wild-type HOXA-AS2 (HOXAAS2-WT) and HOXA3 (HOXA-WT), as well as mutant sequences (HOXA-AS2-MUT and HOXA3MUT), containing the wild-type or mutant putative binding sites of miR-15a-5p were augmented and then cloned into pmIRGLO-control luciferase reporter vectors (Promega, Madison, WI). The reporter vectors with miR-15a-5p mimics and controls (miR-NC) were co-transfected into K-1 and BHP2-7 cells with Lipofectamine 2000 (Invitrogen) for $36 \mathrm{~h}$. Because HOXA-AS2 were highly expressed in K-1 and BHP2-7 cells, we selected the two cells for subsequent analysis. Afterward, cells were collected and lysed, and luciferase activity was tested with a Dual-Luciferase ${ }^{\circledR}$ Reporter Assay System (Promega).

\section{Subcellular fractionation assay}

Cytoplasmic and nuclear fractions were extracted from PTC cell lines using NE-PER Nuclear and Cytoplasmic Extraction Reagents (Thermo Fisher Scientific). $1 \times 10^{7} \mathrm{~K}-1$ and BHP2-7 cells were first washed twice in pre-chilled PBS and lysed in $500 \mu \mathrm{l}$ of cell fractionations buffer containing NP40 (10 mM HEPES, $60 \mathrm{mM} \mathrm{KCl,} 1 \mathrm{mM}$ EDTA, $0.075 \%(\mathrm{v} / \mathrm{v}) \mathrm{NP} 40,1 \mathrm{mM}$ DTT and $1 \mathrm{mM}$ PMSF, $\mathrm{pH}$ 7.6). After incubated on ice for $3 \mathrm{~min}$ utes, lysates were centrifuged at $1500 \mathrm{~g}$ for $4 \mathrm{~min}$ utes. The nuclear and cytoplasmic cell fractions were separated. The cytoplasm supernatant was transferred into a fresh RNase-free tube. Nuclear fractions were cultured in $100 \mu \mathrm{l}$ of nuclear lysis buffer containing $20 \mathrm{mM}$ tris- $\mathrm{HCl}, 420 \mathrm{mM} \mathrm{NaCl}$, $1.5 \mathrm{mM} \mathrm{MgCl} 2,0.2 \mathrm{mM}$ EDTA, $1 \mathrm{mM}$ PMSF and $25 \%$ (v/v) glycerol, $\mathrm{pH}$ 8.0. After incubation at $4^{\circ} \mathrm{C}$ for an hour, nuclear extracts were reaped through centrifugation at $12,000 \mathrm{~g}$ for 10 minutes. RNAs derived from the cytoplasmic or nuclear extracts were obtained using TRIzol (Life Technologies, Carlsbad, CA, USA). Followed by elution, isolated RNAs were analyzed by qRT-PCR. The levels of nucleus control (U6), cytoplasm control (GAPDH) and HOXA-AS2 were separately measured.

\section{RNA-binding protein immunoprecipitation assay}

For RNA-binding protein immunoprecipitation (RIP) assays, PTC cell lines were co-transfected with pcDNA-MS2, pcDNA-HOXA-AS2-MS2, and pBobi-MS2-GFP. The RIP assay was conducted utilizing an anti-green fluorescent protein antibody (Abcam, Cambridge, United Kingdom) and MagnaRIP RIP kit (Millipore, Bedford, MA) according to the manufacturer's instructions. Immunoglobulin G (Millipore) was used as a negative control (input group).

\section{Western blot}

Radioimmunoprecipitation assay buffer was used to extract all proteins from cells, and a NanoDrop 2000 spectrophotometer (Thermo Fisher Scientific) was used to determine the concentration of proteins in each sample. To prepare protein samples, we treated cells thrice with $3 \mathrm{ml}$ of precooled 0.01M PBS. After removing PBS, cells were incubated with $1 \mathrm{ml}$ RIPA lysis buffer on ice for half an hour. Thereafter, cells were centrifuged at $12000 \mathrm{rpm}$ at $4^{\circ} \mathrm{C}$ for 5 minutes. At length, the supernatant was transferred into $0.5 \mathrm{~min}$ centrifugal tube, followed by preservation at $-20^{\circ} \mathrm{C}$. Proteins $(50 \mu \mathrm{g} /$ lane $)$ were separated by sodium dodecyl sulfate-polyacrylamide gel electrophoresis and then transferred onto polyvinylidene fluoride membranes (Millipore). Membranes were blocked with $5 \%$ bovine serum albumin in Tris-buffered saline with $0.1 \%$ Tween 20 (TBST) for $2 \mathrm{~h}$ at room temperature prior to incubation with primary antibodies at $4^{\circ} \mathrm{C}$ overnight. Primary antibodies used were anti-HOXA3 (1:1,000; Santa Cruz Biotechnology, Santa Cruz, CA), anti- $\beta$-catenin (1:1,000; Cell Signaling Technology, Danvers, MA), anti-cyclin D1 (1:1,000; Cell Signaling Technology), anti-c-Myc (1:1,000; Cell Signaling Technology), and anti-GAPDH (1:5,000; Sigma-Aldrich). Then, membranes were washed in TBST and incubated with a horseradish peroxidase-tagged secondary antibody at $37^{\circ} \mathrm{C}$ for $1 \mathrm{~h}$. Afterward, membranes were again washed with TBST, and protein bands of interest were detected by an enhanced chemiluminescence system (Pierce, Rockford, IL). Bands were 

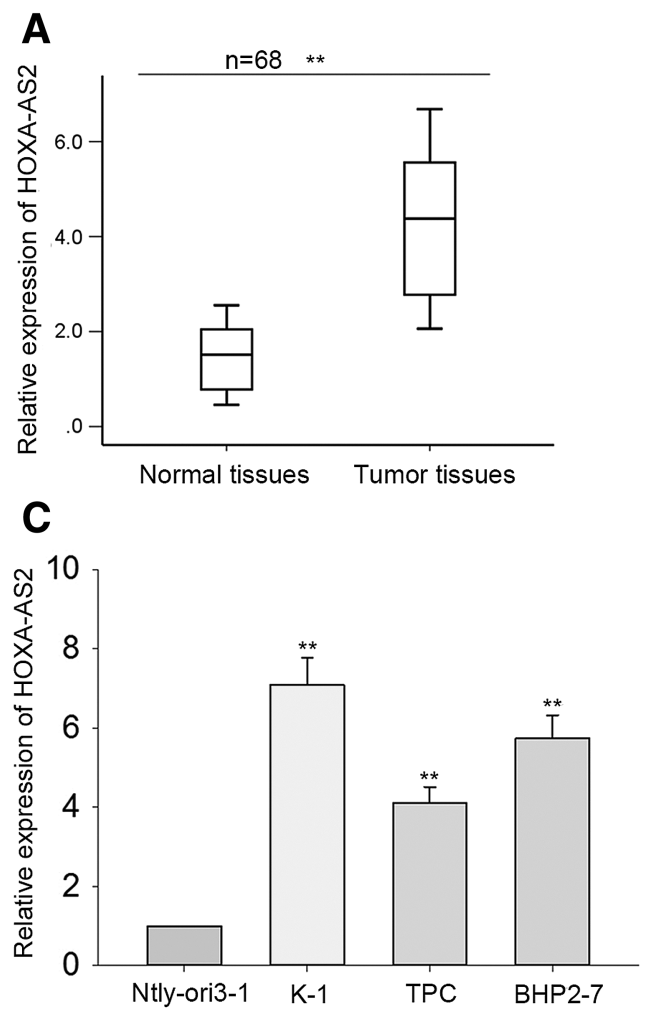

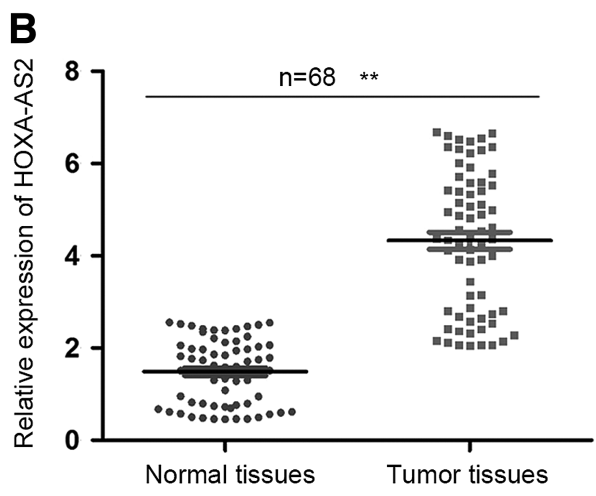

D

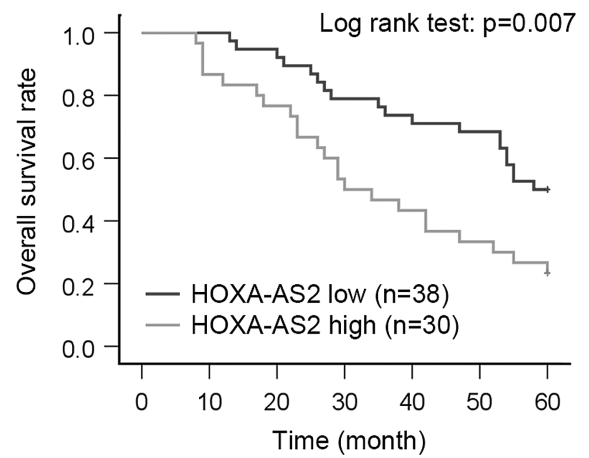

Figure 1. Higher expression of HOXA-AS2 is associated with a poor prognosis in papillary thyroid cancer (PTC) patients. (A-C) Quantitative real-time polymerase chain reaction (qRT-PCR) analysis revealed that expression of HOXA-AS2 in PTC tissues and cell lines was higher than that in adjacent normal tissues and a normal human thyroid epithelial cell line. (C) The Kaplan-Meier method showed that HOXA-AS2 expression was negatively related to the overall survival rate of 68 PTC patients. Error bars represent the mean \pm standard deviation of at least three independent experiments. ${ }^{* *} p<0.01$ versus control group.

quantified by ImageJ software (National Institutes of Health, Bethesda, MD).

\section{In vivo assays}

6-week-old female nude mice were bought from National Laboratory Animal Center (Beijing, China). Mice were first maintained in the SPFgrade pathogen-free animal laboratory equipped with laminar flow equipment and $12 \mathrm{~h}$ light-dark cycles. Nude mice were free to autoclaved food and water. All experiments with mice were conducted strictly in accordance with the Guide for the Care and Use of Laboratory Animals. In vivo assay was approved by the review board of the State key laboratory of environmental microorganism utilization and safety control at Shaoxing Hospital of China Medical University (Shaoxing, P.R. China). $5 \times 10^{4} \mathrm{~K}-1$ cells were transfected with various plasmids or combinations. Cells were reaped and subcutaneously injected into the left flank of the nude mice using sterile 28-gauge needles. One week after injection, mice were randomly divided into two groups ( $\mathrm{n}=4$ per group) when PTC tumors had reached approximately $3 \mathrm{~mm}^{3}$. Tumor volume was measured every fourth day. Four weeks after implantation, all mice were sacrificed. Tumors were excised and weighed for subsequent analysis. The tumor sizes were determined by measuring their length (l) and width (w) via a digital caliper. The tumor volumes (V) were calculated with the following equation: $\mathrm{V}=\mathrm{lw} 2 / 2$. Finally, we obtained approximately $8 \times 10^{3} \mathrm{~mm}^{3}$ PTC tumors.

\section{Bioinformatics analysis}

The University of California-Santa Cruz genome database (http://genome.ucsc.edu) was applied to the potential downstream gene of HOXA-AS2. The binding sites between HOXA-AS2 and miR-15a-5p were predicted by DIANA tools.* The potential binding sequences of miR-15a-5p in HOXA3 3'UTR was acquired from TargetScan. ${ }^{\dagger}$

\footnotetext{
*http://carolina.imis.athena-innovation.gr/diana_tools/web/index .php?r=lncbasev2\%2Findexpredicted

'www.targetscan.org/mamm_31/
} 


\section{Statistical analysis}

All results are shown as means \pm standard deviations from three independent experiments. Student's $t$-test was applied to measure the differences between two groups. One-way analysis of variance was performed to compare differences among more than two groups. Statistical analyses were completed using IBM SPSS Statistics for Windows v19.0 (IBM Corp., Armonk, NY). Statistically significant correlations between HOXA-AS2 and miR-15a-5p, as well as miR15a-5p and HOXA3, were analyzed using Spearman's correlation analysis. Differences were considered statistically significant with a $p$-value of $<0.05$.

\section{RESULTS}

\section{Heightened expression of HOXA-AS2 \\ is associated with a poor prognosis \\ in PTC patients}

qRT-PCR results showed HOXA-AS2 expression levels in PTC tissues and cell lines were obviously higher than that in matched normal tissues and control cells (Fig. 1A-C). Using the mean HOXAAS2 expression value as a threshold, PTC samples were classified into high and low HOXA-AS2 expression groups. Next, the correlation between HOXA-AS2 expression and clinicopathologic features of PTC patients was analyzed. As presented in Table 1, dysregulation of HOXA-AS2 indicated advanced tumor-node-metastasis staging and lymph

Table 1. Correlation between HOXA-AS2 expression and clinical features of PTC patients $(n=68)$

\begin{tabular}{|c|c|c|c|}
\hline \multirow[b]{2}{*}{ Variable } & \multicolumn{2}{|c|}{ HOXA-AS2 expression } & \multirow[b]{2}{*}{$\mathrm{p}$} \\
\hline & Low & High & \\
\hline \multicolumn{4}{|l|}{ Age (years) } \\
\hline$<45$ & 16 & 17 & \multirow[t]{2}{*}{0.233} \\
\hline$\geq 45$ & 22 & 13 & \\
\hline \multicolumn{4}{|l|}{ Sex } \\
\hline Male & 25 & 9 & \multirow[t]{2}{*}{$0.003^{* *}$} \\
\hline Female & 13 & 21 & \\
\hline \multicolumn{4}{|c|}{ Extrathyroidal extension } \\
\hline Negative & 36 & 26 & \multirow[t]{2}{*}{0.244} \\
\hline Positive & 2 & 4 & \\
\hline \multicolumn{4}{|l|}{ Tumor size } \\
\hline$\leq 1$ & 25 & 12 & \multirow[t]{2}{*}{0.34} \\
\hline$>1$ & 13 & 18 & \\
\hline \multicolumn{4}{|l|}{ TNM stage } \\
\hline$|/| \mid$ & 34 & 15 & \multirow[t]{2}{*}{$<0.001^{* * *}$} \\
\hline III/IV & 4 & 15 & \\
\hline \multicolumn{4}{|c|}{ Lymph node metastasis } \\
\hline Negative & 23 & 9 & \multirow[t]{2}{*}{$0.012^{*}$} \\
\hline Positive & 15 & 21 & \\
\hline \multicolumn{4}{|c|}{ Nodular goiter } \\
\hline Negative & 4 & 9 & \multirow[t]{2}{*}{$0.043^{*}$} \\
\hline Positive & 34 & 21 & \\
\hline
\end{tabular}

Low/high by the sample median. Pearson's chi-square test. ${ }^{*} p<0.05$; ${ }^{* *} p<0.01 ;{ }^{* * *} p<0.001$.

PTC, papillary thyroid cancer; TNM, tumor-node-metastasis. node metastasis. According to Kaplan-Meier analysis, patients with higher HOXA-AS2 expression had a lower survival rate than those with lower expression ( $p=0.007$; Fig. 1C). These findings indicate a potential oncogenic role for HOXA-AS2 in PTC.

\section{HOXA-AS2 knockdown suppressed cell proliferation and accelerated apoptosis}

In order to assess HOXA-AS2 function in PTC, K-1 and BHP2-7 cell lines were transfected with si-HOXA-AS2\#1 and si-HOXA-AS2\#2 or si-NC (Fig. 2A). MTT assay results revealed that the proliferation of cells transfected with either siHOXA-AS2\#1 or si-HOXA-AS2\#2 was greatly suppressed (Fig. 2B). Moreover, flow cytometry results showed that knockdown of HOXA-AS2 remarkably enhanced the apoptosis rate of PTC cells (Fig. 2C).

\section{Knockdown of HOXA-AS2 inhibited migration and invasion of PTC cells}

To probe into the effect HOXA-AS2 depletion on the migratory and invasive abilities of K-1 and BHP2-7 cells, we carried out Transwell ${ }^{\circledR}$ assays. Results revealed that HOXA-AS2 knockdown significantly reduced the number of both migratory and invasive cells, indicating the negative impact of HOXA-AS2 knockdown on PTC cell migration and invasion (Fig. $3 \mathrm{~A}$ and $\mathrm{B}$ ).

\section{Knockdown of HOXA3 suppressed PTC cell proliferation, migration, and invasion but induced apoptosis}

According to an online search of the University of California-Santa Cruz genome database (http:// genome.ucsc.edu), HOXA3 is a potential downstream gene of HOXA-AS2. Therefore, their potential correlation in PTC progression was investigated herein. Consistent with HOXA-AS2 results reported above, HOXA3 expression was upregulated in both PTC tissues and cell lines (Fig. 4A). Hence, HOXA-AS2 expression and HOXA3 expression were positively correlated (Fig. 4B). MTT assessment of HOXA3 function in PTC cells co-transfected with si-HOXA3\#1 and si-HOXA3\#2 revealed that HOXA3 silencing markedly inhibited cellular proliferation (Fig. 4D) but increased apoptosis rates (Fig. 4E). Moreover, Transwell ${ }^{\circledR}$ assays showed that knockdown of HOXA3 evidently lessened the migratory and invasive ability of PTC cells. These results indicate an oncogenic role for HOXA3 in PTCs.

\section{HOXA-AS2 is a molecular sponge of miR-15a-5p}

Previous studies have shown that lncRNAs can act as ceRNAs to upregulate mRNAs by sponging 

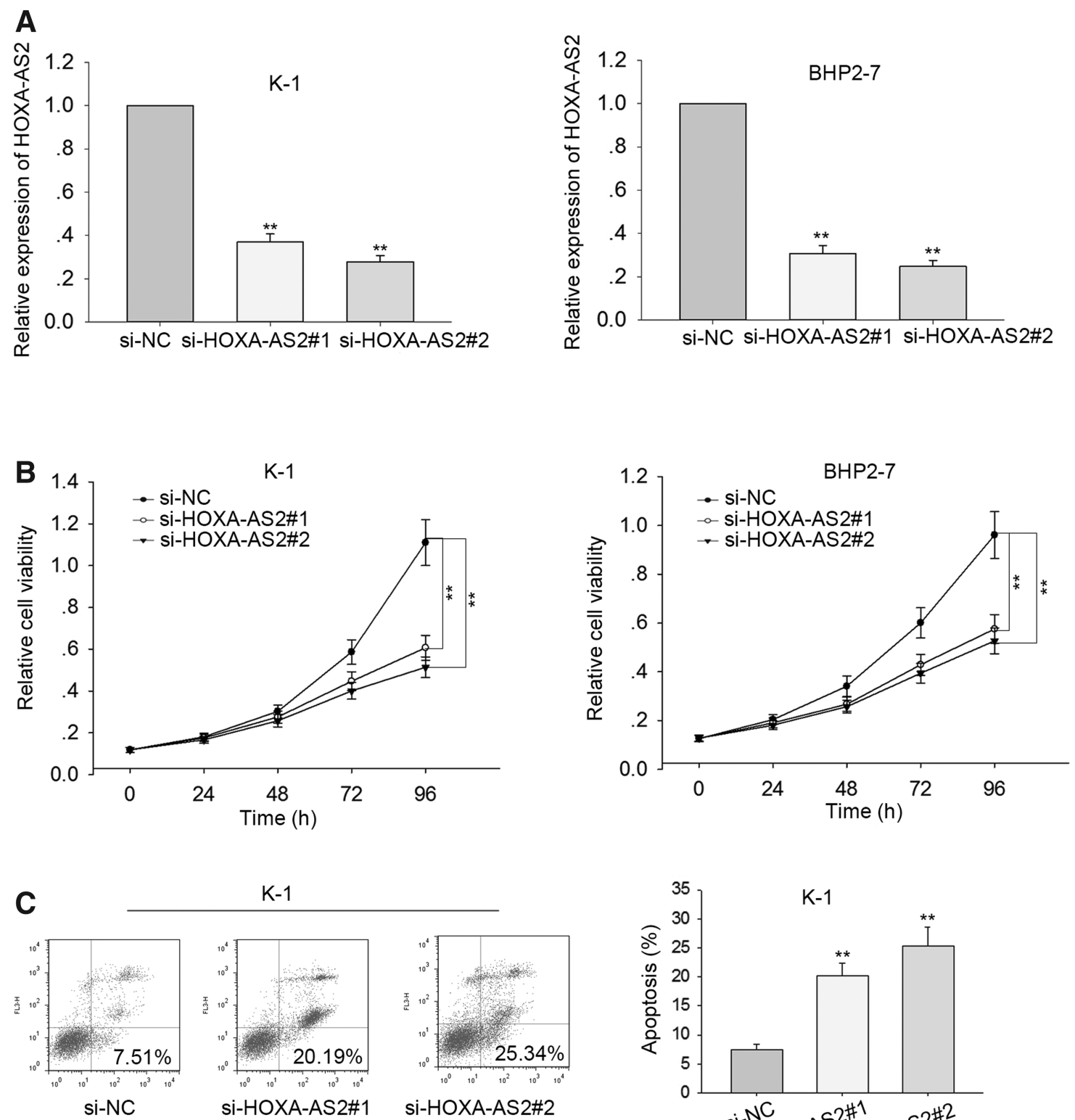

$\mathrm{K}-1$
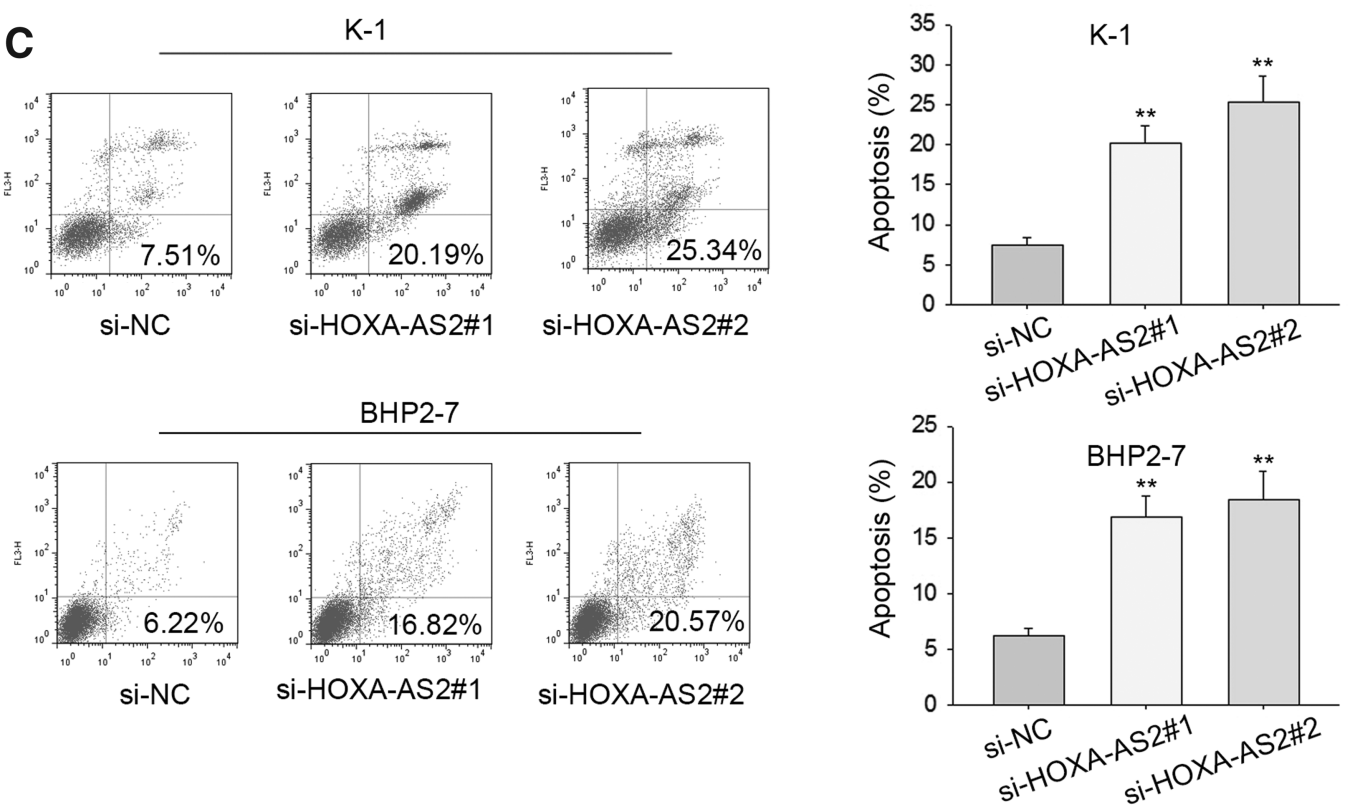

Figure 2. Knockdown of HOXA-AS2 suppressed cell proliferation and accelerated apoptosis. (A) qRT-PCR detected the transfection efficiency of si-HOXAAS2\#1 and si-HOXA-AS2\#2 into PTC cells. (B) A MTT assay was used to explore the effect of HOXA-AS2 silencing on cell viability. (C) Flow cytometry analysis of apoptosis in PTC cells transfected with si-HOXA-AS2\#1 and si-HOXA-AS2\#2. Error bars represent the mean \pm standard deviation of at least three independent experiments. ${ }^{* *} p<0.01$ versus control group. 


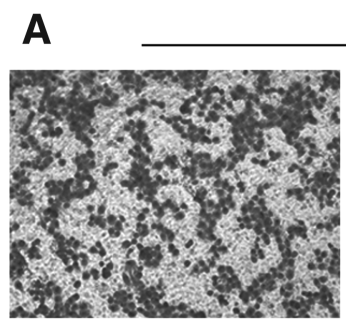

si-NC
$\mathrm{K}-1$

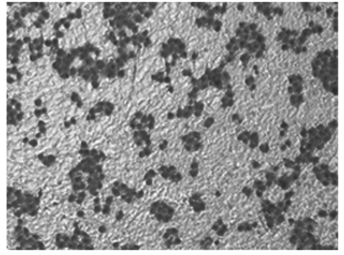

si-HOXA-AS2\#1

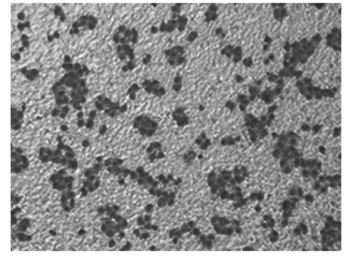

si-HOXA-AS2\#2

BPH2-7

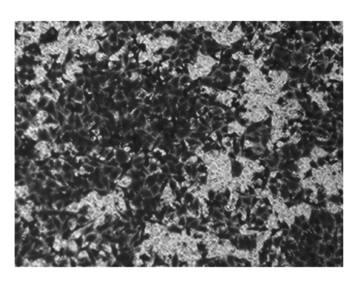

si-NC

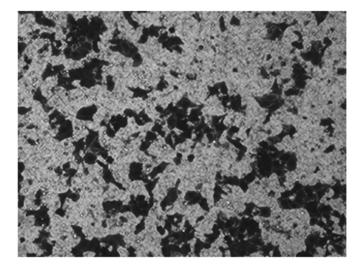

si-HOXA-AS2\#1

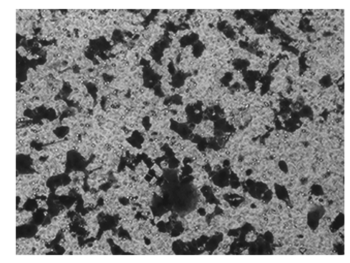

si-HOXA-AS2\#2

$\mathrm{K}-1$

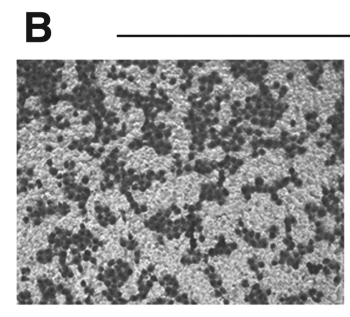

si-NC

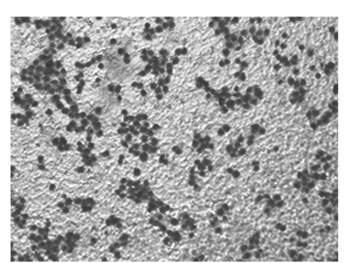

si-HOXA-AS2\#1

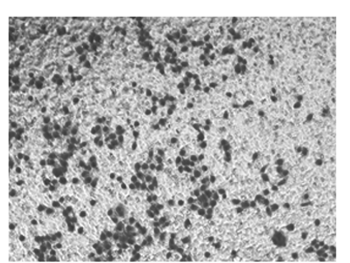

si-HOXA-AS2\#2

BPH2-7

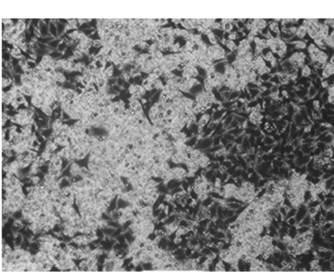

si-NC

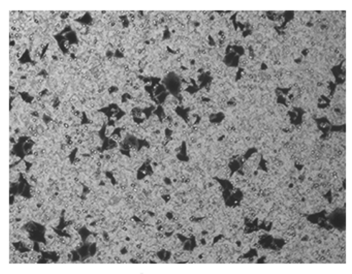

si-HOXA-AS2\#1

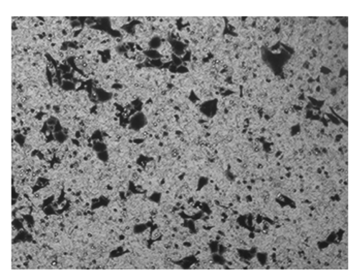

si-HOXA-AS2\#2
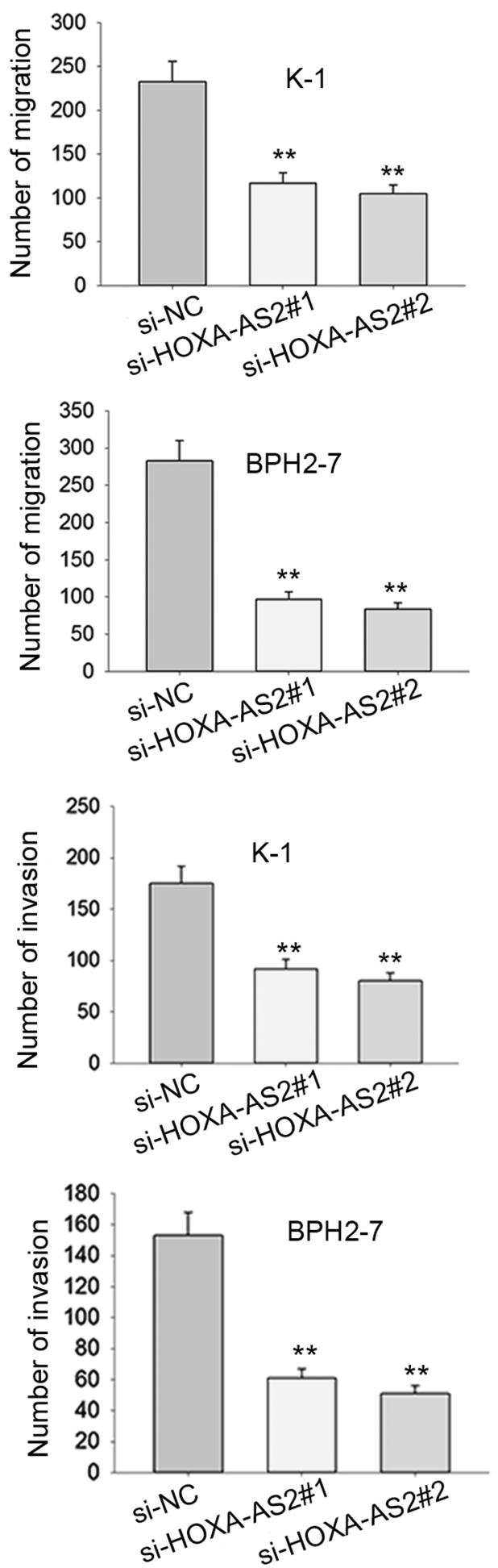

Figure 3. Silencing of HOXA-AS2 inhibited migration and invasion of PTC cells. (A and B) Transwell ${ }^{\circledR}$ assays were applied to examine the effects of HOXAAS2 silencing on the migration and invasion of PTC cells. Error bars represent the mean \pm standard deviation of at least three independent experiments. ${ }^{*} p<0.01$ versus control group.

miRNAs. ${ }^{32-34}$ Based on the above findings, HOXA-AS2 was hypothesized to act as a ceRNA to upregulate HOXA3 in PTC. Subcellular fractionation assay results showed that the level of cytoplasmic HOXA-AS2 in PTC cells was greater than that in the nucleus (Fig. 5A), suggesting that HOXA-AS2 lncRNA regulates gene expression in PTC cells at the post-transcriptional level. Subsequently, 14 miRNAs that can bind HOXA3 were found using bioinformatics analysis software 


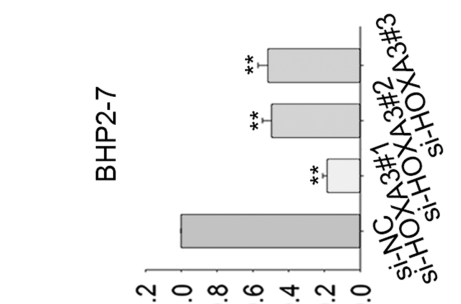

$\varepsilon \forall \mathrm{XOH}$ 10 uolssaldxə

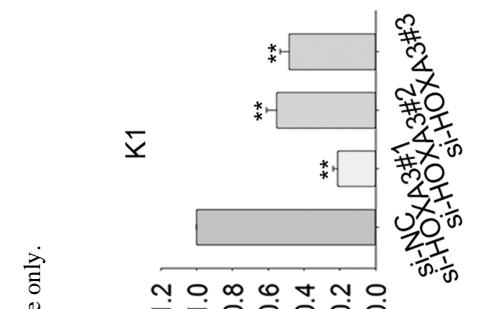

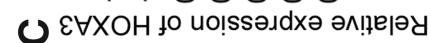

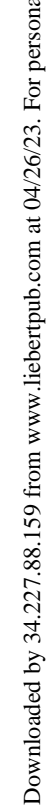

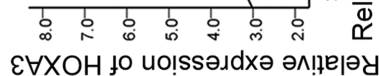

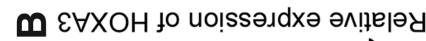

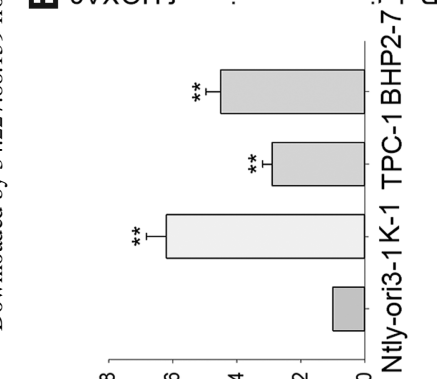

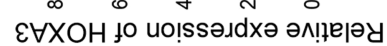

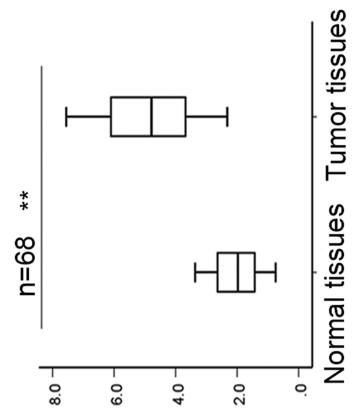

$\varangle \varepsilon \forall X O H$ tо ио!ssәлdхә әм!ฺе|әу
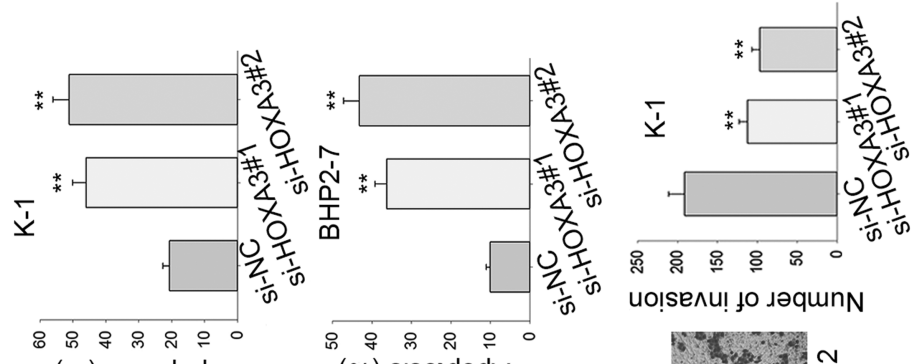
(\%) s!soldod $\forall$
(\%) s!̣oldod $\forall$

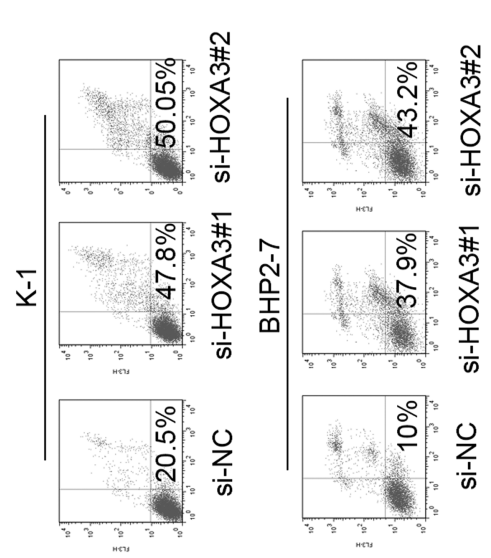

Ш
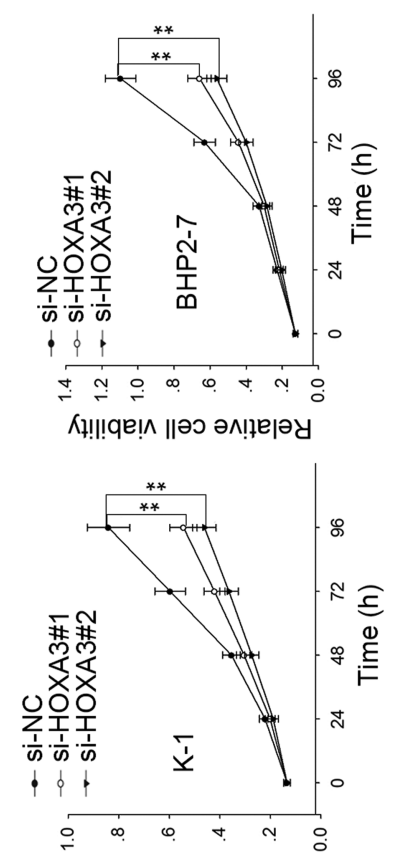

Кํ!!!qe!^ ||әว әл!ฺе|әу

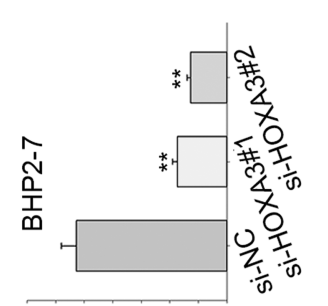

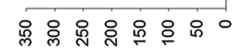
uo!senu! fo ıəqunN

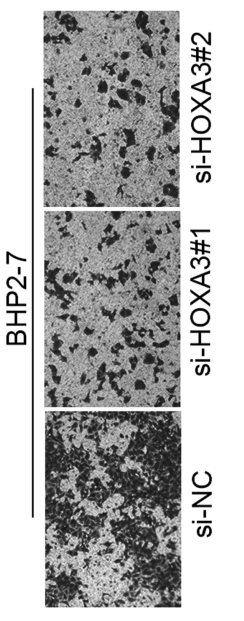

$\circlearrowleft$
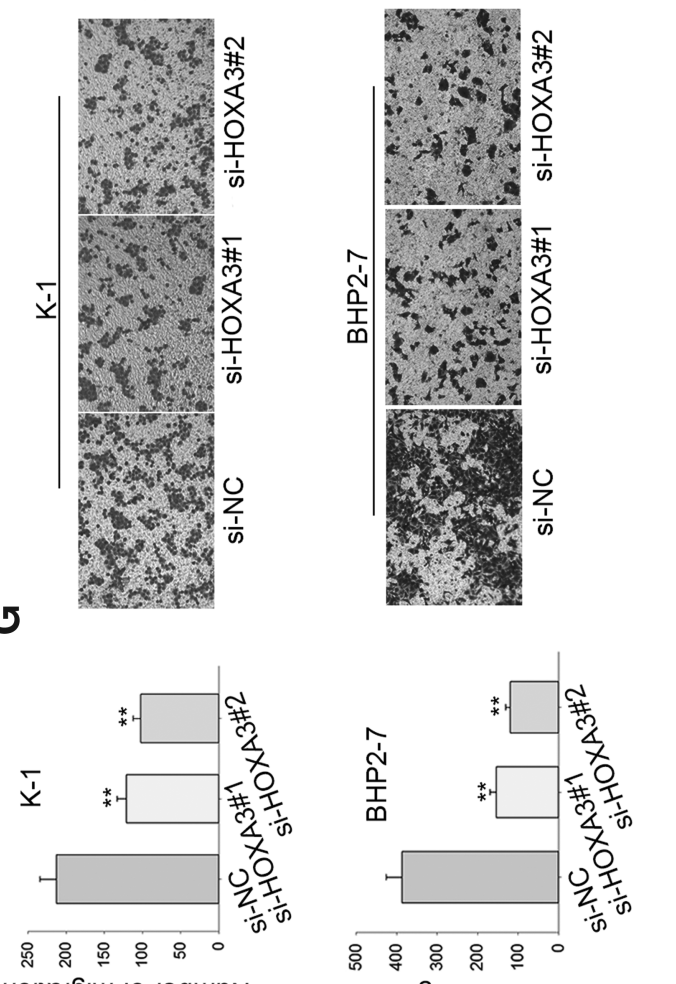

uo!̣ej6!iu jo jəqunn
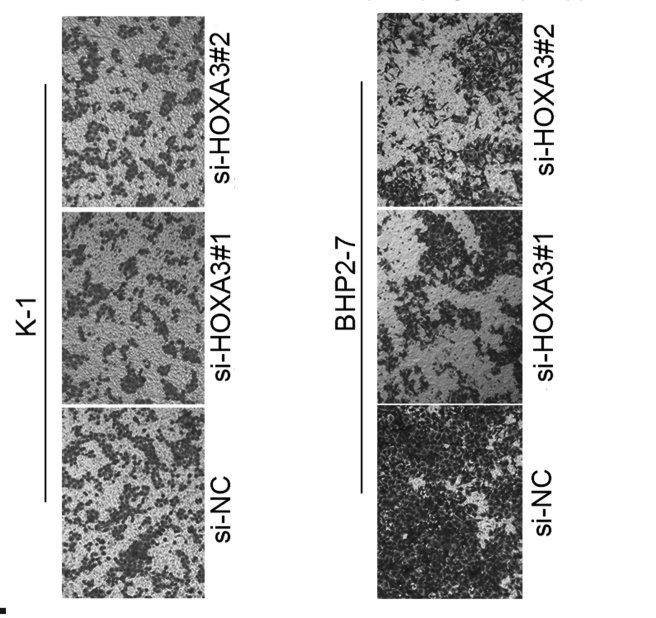

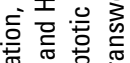

뜬 흥

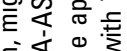

言变步

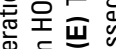

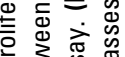

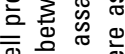

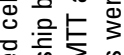

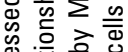

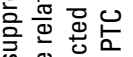

空势

安造造

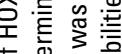

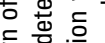

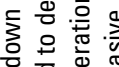

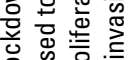

产

$+3 \overline{0}$

은 $\frac{0}{2}$ 
A

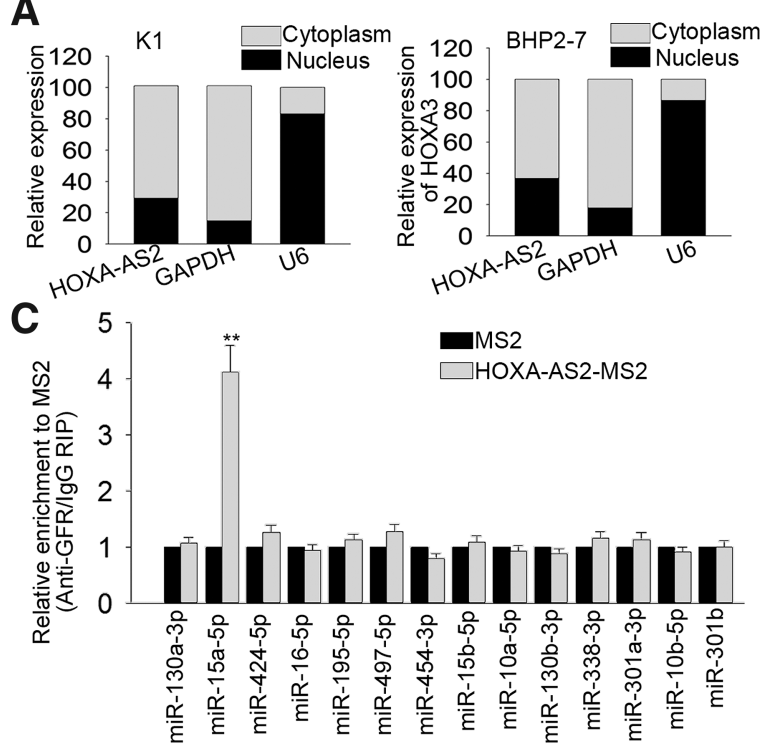

D

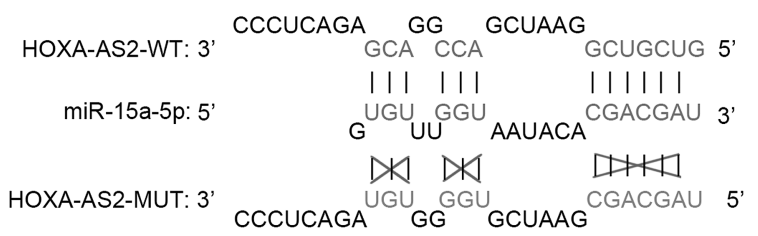

B

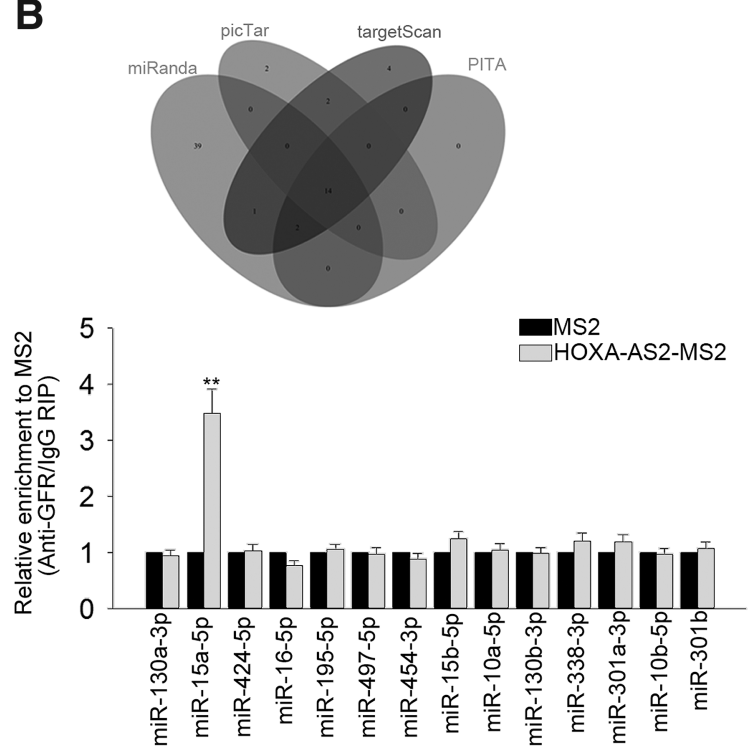

E
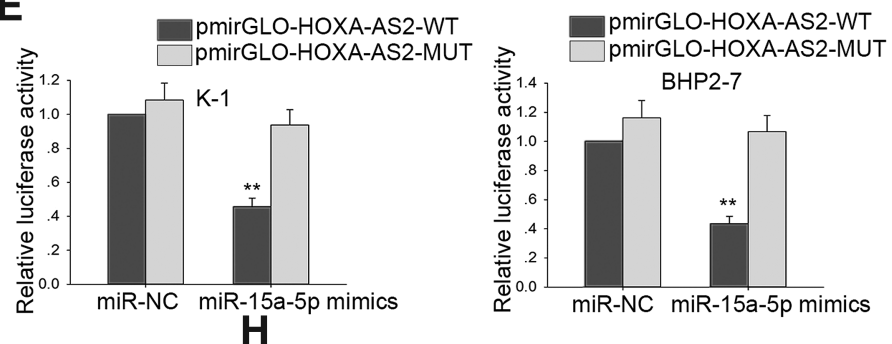

H
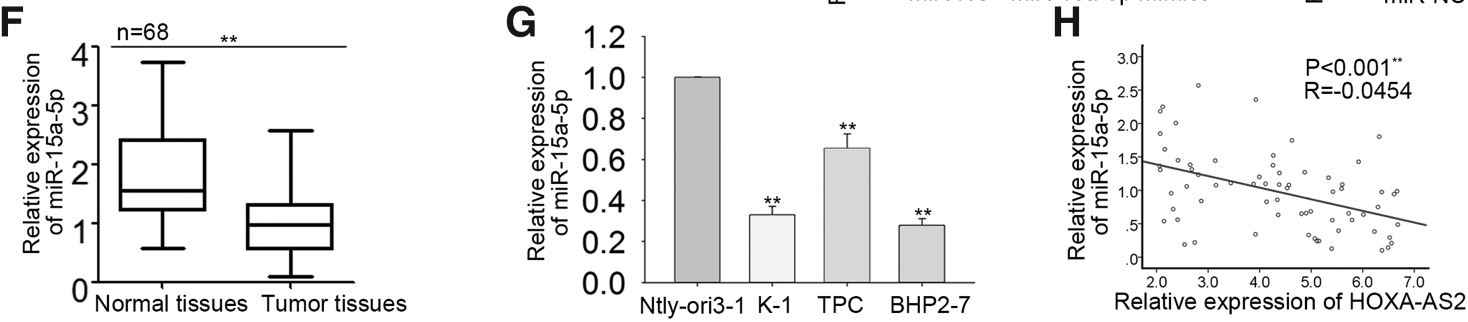

Figure 5. HOXA-AS2 is a molecular sponge of miR-15a-5p. (A) Subcellular fractionation assay was utilized to identify the location of HOXA-AS2 in PTC cells. (B) Four bioinformatics prediction tools (miRanda, picTar, TargetScan, and PITA) were used to predict 14 miRNAs that bind HOXA3. (C) RNA-binding protein immunoprecipitation assays were carried out in two PTC cell lines to detect which miRNA could bind HOXA-AS2. (D) The binding sequence between HOXAAS2 and miR-15a-5p. (E) Dual-Luciferase ${ }^{\circledR}$ reporter assay was performed to validate the interaction between HOXA-AS2 and miR-15a-5p. (F and $\left.\mathbf{G}\right)$ The levels of miR-15a-5p were measured in PTC tissues and cell lines by qRT-PCR. (H) The association between HOXA-AS2 and miR-15a-5p was identified by Spearman's correlation analysis. Error bars represent the mean \pm standard deviation of at least three independent experiments. ${ }^{* *} p<0.01$ versus control group.

(miRanda, picTar, TargetScan, and PITA; Fig. 5B). A RIP assay was then used to determine whether these 14 miRNAs can also bind HOXA-AS2 in PTC cells. The results showed co-immunoprecipitation of HOXA-AS2 and miR-15a-5p from BHP2-7 and $\mathrm{K}-1$ cell lines (Fig. 5C). Next, HOXA-AS2 and miR15a-5p binding sites were predicted using DIANA tools (http://carolina.imis.athena-innovation.gr/ diana_tools/web/index.php? $r=\operatorname{lncbasev2\% 2Findex-~}$ predicted; Fig. 5D) and confirmed by DualLuciferase ${ }^{\circledR}$ reporter assay. As shown in Fig. 5E, the luciferase activity of HOXA-AS2-WT was decreased by the miR-15a-5p mimics but not miR-NC. More- over, miR-15a-5p was found to be downregulated in both PTC tissues and cell lines (Fig. 5F and G). Finally, Spearman's correlation analysis revealed a negative association between HOXA-AS2 and miR15a-5p expression (Fig. 5H). Taken together, these results indicate miR-15a-5p is a target gene of HOXA-AS2.

\section{HOXA-AS2 contributed to HOXA3 upregulation by competitively binding miR-15a-5p}

The putative binding sequence between miR15a-5p and HOXA3 was obtained from Target- 
A

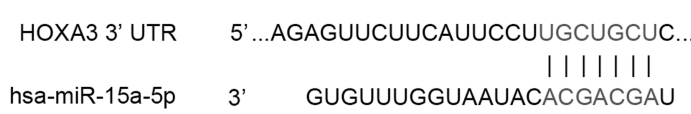

HOXA3 3' UTR-MUT 5' ...AGAGUUCUUCAUUCCUACGACGAC..
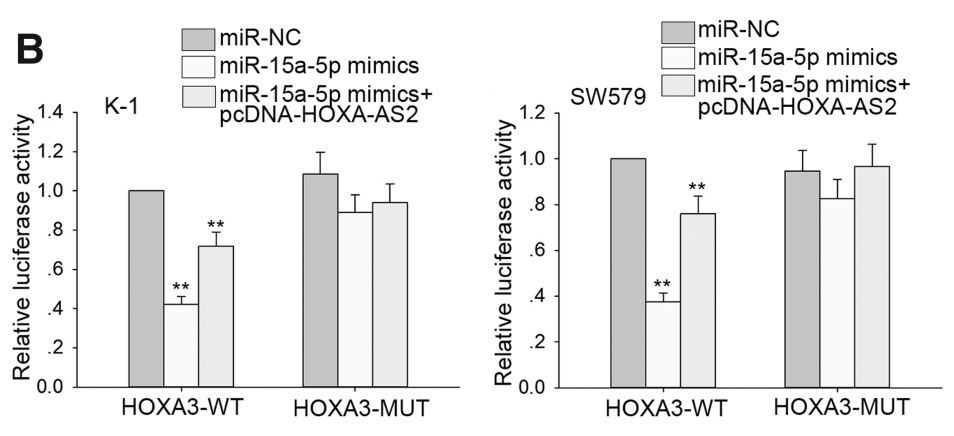
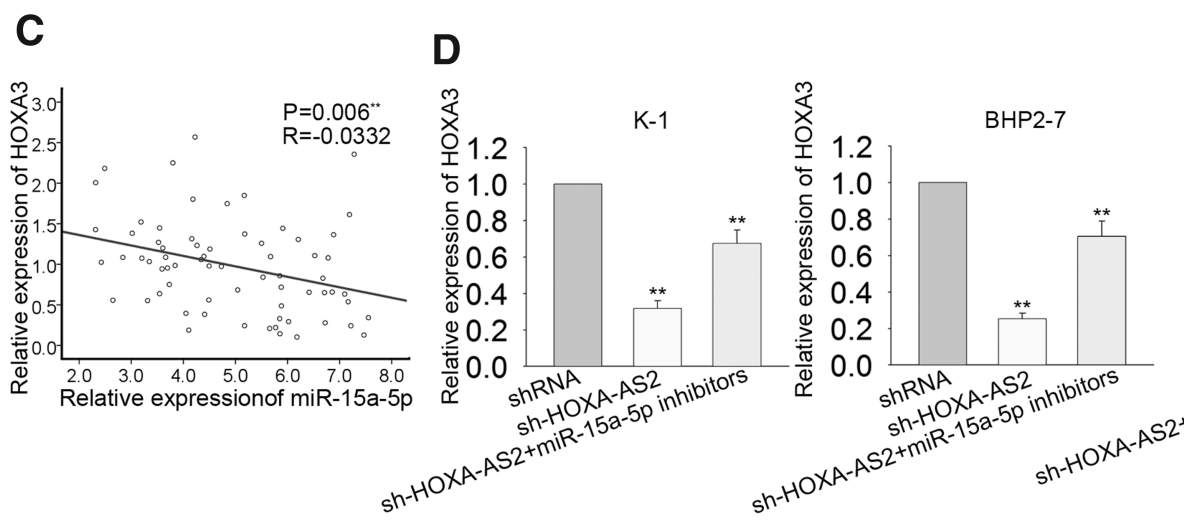

$\mathbf{E}$

Figure 6. HOXA-AS2 contributed to the upregulation of HOXA3 by competitively binding miR-15a-5p. (A) The putative binding sequence between miR-15a-5p and HOXA3 was obtained from TargetScan. (B) Dual-Luciferase ${ }^{\circledR}$ reporter analysis was used to confirm binding of HOXA-AS2 to miR-15a-5p. (C) The association between miR-15a-5p and HOXA3 was analyzed by Spearman's correlation analysis. (D and E) qRT-PCR and Western blot were used to examine HOXA3 mRNA and protein levels, respectively, in PTC cells co-transfected with sh-HOXA-AS2 and miR-15a-5p inhibitors. Error bars represent the mean \pm standard deviation of at least three independent experiments. ${ }^{* *} p<0.01$ versus control group.

$\operatorname{Scan}^{\dagger}$ (Fig. 6A) and confirmed by Dual-Luciferase ${ }^{\circledR}$ reporter assay. Co-transfection of HOXA3-WT with miR-15a-5p mimics reduced luciferase activity in PTC cells. However, activity was recovered by upregulation of HOXA-AS2 (Fig. 6B). Spearman's correlation analysis revealed a negative association between miR-15a-5p and HOXA3 expression (Fig. 6C). Moreover, qRT-PCR and Western blot analyses of PTC cells co-transfected with shHOXA-AS2 and miR-15a-5p inhibitors showed that decreases in HOXA3 mRNA and protein levels caused by sh-HOXA-AS2 could be recovered by miR15a-5p inhibitors (Fig. 6D and E). These findings indicate that HOXA-AS2 positively modulates HOXA3 expression in PTC cells by competitively binding to miR-15a-5p.

\section{HOXA-AS2/miR-15a-5p/HOXA3 axis regulates PTC progression}

Rescue assays were used to demonstrate the role of HOXA-AS2/miR-15a-5p/HOXA3 axis in PTC progression. Results of MTT assays showed that HOXA3 and miR-15a-5p inhibitors partially reversed sh-HOXA-AS2-mediated cell proliferation

\footnotetext{
${ }^{\dagger}$ www.targetscan.org/mamm_31
}

in $\mathrm{K}-1$ cell line (Fig. 7A). Moreover, the increased rate of apoptosis caused by HOXA-AS2 knockdown could be decreased by upregulation of HOXA3 or downregulation of miR-15a-5p (Fig. 7B). Furthermore, Transwell ${ }^{\circledR}$ assays revealed that migration and invasion activity suppressed by sh-HOXA-AS2 could be recovered by HOXA3 overexpression or miR-15a-5p inhibitors (Fig. 7C and D).

\section{HOXA-AS2/miR-15a-5p/HOXA3 axis regulates PTC tumor growth in vivo}

In vivo experiments using mice were conducted to demonstrate the function of the HOXA-AS2/ miR-15a-5p/HOXA3 axis further in PTC tumor growth. As illustrated in Fig. 8A, tumor growth was inhibited by HOXA-AS2 knockdown but promoted by miR-15a-5p knockdown or HOXA3 overexpression. Additionally, the growth inhibition caused by HOXA-AS2 knockdown was partly recovered by miR-15a-5p silencing or HOXA3 overexpression. Moreover, the decreased miR-15a$5 p$ expression or increased HOXA3 expression significantly rescued the sh-HOXA-AS2-mediated reduced tumor volume and weight. These results illustrate regulation of PTC tumor growth in vivo by the HOXA-AS2/miR-15a-5p/HOXA3 axis. 

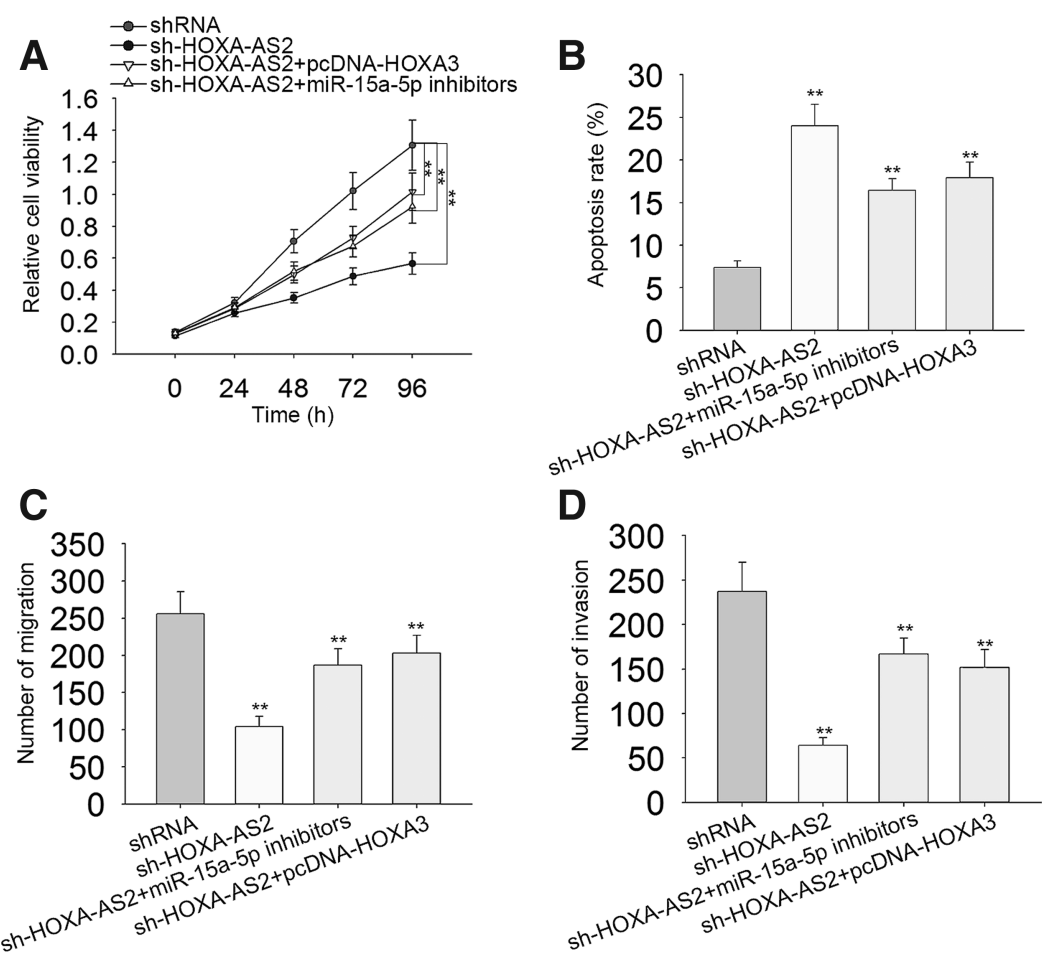

Figure 7. The HOXA-AS2/miR-15a-5p/HOXA3 axis regulates PTC progression. (A) A MTT assay was performed to determine the effect of miR-15a-5p inhibitors or pcDNA-HOXA3 on the viability of PTC cells transfected with sh-HOXA-AS2. (B) Flow cytometry analysis of the apoptosis rate of PTC cells co-transfected with shHOXA-AS2 and miR-15a-5p inhibitors or sh-HOXA-AS2 and pcDNA-HOXA3. (C and D) The migration and invasion abilities of PTC cells were evaluated by Transwell $^{\circledR}$ assays. Error bars represent the mean \pm standard deviation of at least three independent experiments. ${ }^{* *} p<0.01$ versus control group.

\section{HOXA-AS2/miR-15a-5p/HOXA3 axis synergistically regulates Wnt/ $\beta$-catenin signaling}

According to previous studies, Wnt/ $\beta$-catenin signaling plays a crucial role in lncRNA-mediated tumor progression. Based on the above findings, the HOXA-AS2/miR-15a-5p/HOXA3 axis was hypothesized to regulate $\mathrm{Wnt} / \beta$-catenin signaling in
PTC cells. Using Western blot, the levels of three core proteins in the $\mathrm{Wnt} / \beta$-catenin signaling pathway ( $\beta$-catenin, c-Myc, and cyclin D1) were examined. The results showed that knockdown of HOXA-AS2 decreased the levels of all three of these proteins (Fig. 9). However, their levels could be recovered by knockdown of miR-15a-5p or overexpression of HOXA3 (Fig. 9). These results suggest
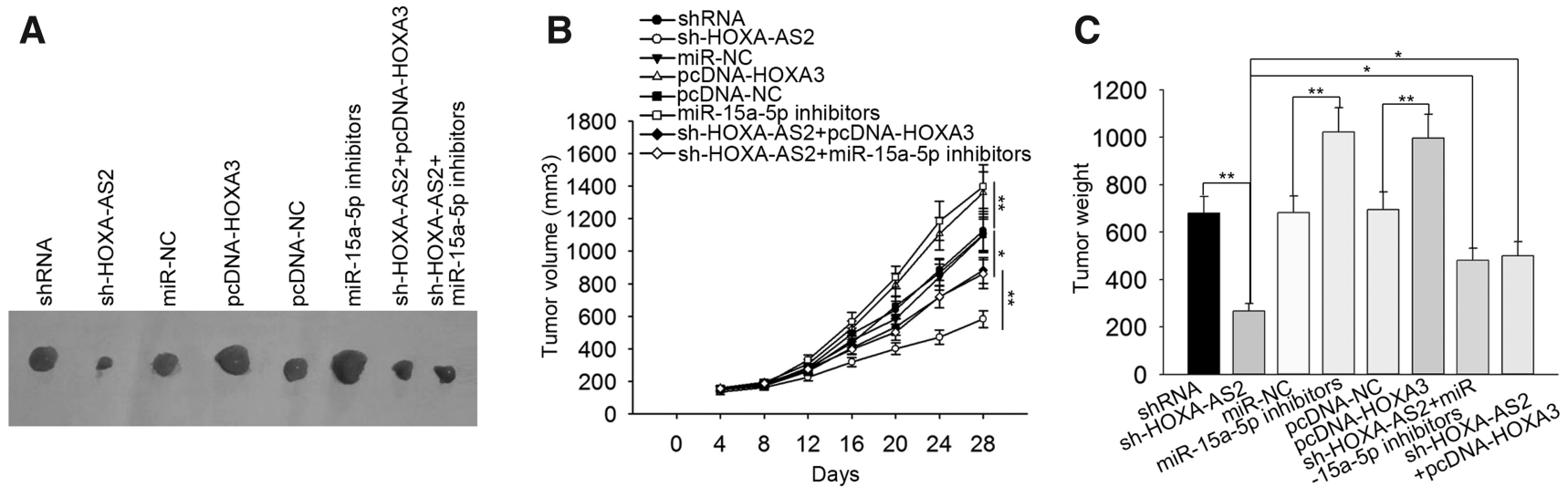

Figure 8. The HOXA-AS2/miR-15a-5p/HOXA3 axis regulates PTC cell growth in vivo. (A) Processed PTC cells were implanted into nude mice, and tumor growth was observed. (B and C) Tumor volume and weight were measured 1 month after implantation of PTC cells into nude mice ( $n=4$ per group). Error bars represent the mean \pm standard deviation of at least three independent experiments. ${ }^{*} p<0.05$ and ${ }^{* *} p<0.01$ versus control group. 

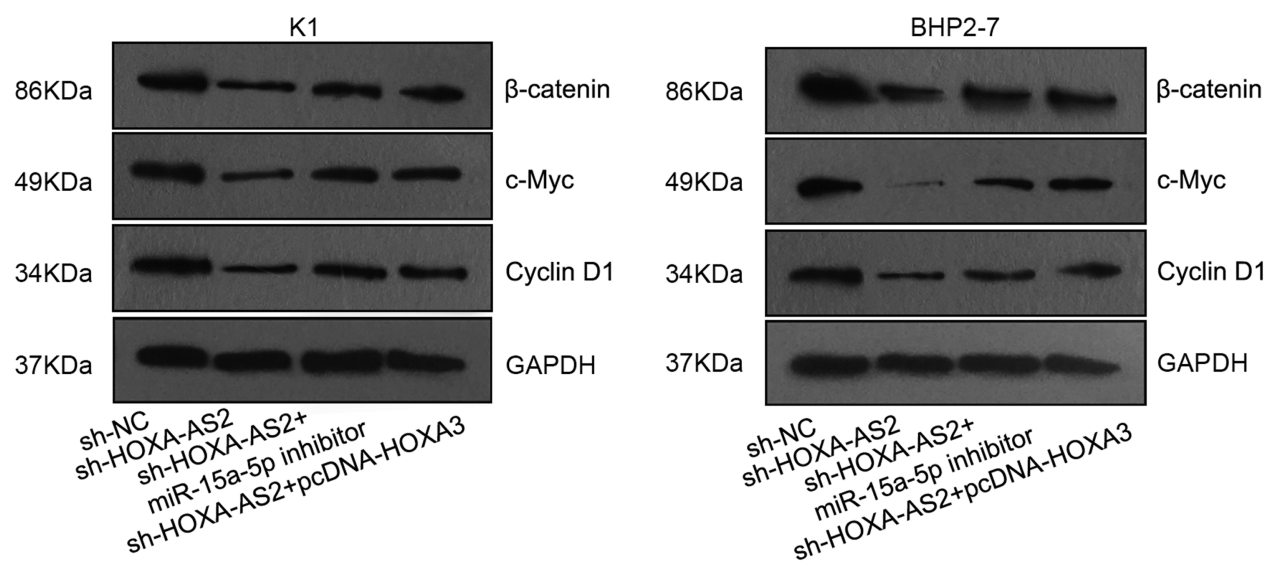

Figure 9. The HOXA-AS2/miR-15a-5p/HOXA3 axis synergistically regulates the Wnt/ $\beta$-catenin signaling pathway. The protein levels of $\beta$-catenin, $\mathrm{c}-\mathrm{Myc}$, and cyclin D1 were examined in PTC cells co-transfected with sh-NC, sh-HOXA-AS2, miR-15a-5p inhibitors, and pcDNA-HOXA3. Error bars represent the mean \pm standard deviation of at least three independent experiments.

the HOXA-AS2/miR-15a-5p/HOXA3 axis modulates PTC progression possibly by regulating Wnt/ $\beta$-catenin signaling proteins.

\section{DISCUSSION}

Thyroid cancer is a common type of human cancer. ${ }^{35}$ Although surgical resection is typically an efficient treatment for thyroid tumors, the 5year survival rate is still bleak, ${ }^{36}$ and many patients die of this disease. ${ }^{37}$ Therefore, it is critical to find more novel therapeutic targets for thyroid cancer. As the most common subtype, PTC was the focus of the present study. Recently, lncRNAs attract increasing attention of researchers, due to their contribution to initiation and development of PTC. With that, we aimed to probe into the novel lncRNA-mediated molecular mechanism in PTC. Currently, we focused on the functional and mechanistic implication of HOXA-AS2 in PTC.

LncRNAs are known to regulate various genes that are crucial for the initiation of tumors ${ }^{38}$ and have oncogenic functions in various malignant tumors. ${ }^{39-43}$ In particular, lncRNA HOXA-AS2 has been shown to act as a tumor promoter in hepatocellular carcinoma, ${ }^{44}$ pancreatic cancer, ${ }^{18}$ and colorectal carcinoma. $^{21}$ However, it is unclear whether HOXA-AS2 is involved in PTC progression. In the present study, we first found the significant upregulation of HOXA-AS2 in PTC tissues and cell lines. Kaplan-Meier method revealed the negative relevance between HOXA-AS2 upregulation and overall survival of PTC patients, implying the potential involvement of HOXA-AS2 in PTC progression. Results from loss-of-function assays indicated that HOXA-AS2 positively regulated PTC cell proliferation, apoptosis, migration and invasion, acting as an oncogene. In mechanism, we observed that HOXA-AS2 could promote HOXA3 expression in PTC cells. Further functional assays demonstrated that HOXA3 exerted oncogenic role in PTC, consistently with HOXA-AS2. Many previous studies have reported that lncRNAs can upregulate mRNAs by acting as ceRNAs. ${ }^{45-47}$ Influenced by this notion, we speculated that HOXA-AS2 might act as a ceRNA in PTC to upregulate HOXA3. Using bioinformatics analysis and mechanistic experiments, it was observed that HOXA-AS2 functioned as a ceRNA via sponging miR-15a-5p to release HOXA3 expression in PTC. Besides, the positive impact of HOXA-AS2-miR-15a-5p-HOXA3 axis on PTC cellular process and tumor growth was validated by rescue assays and in vivo experiments.

The Wnt/ $\beta$-catenin signaling pathway has been shown to be a critical element in the tumorigenesis of human tumors. ${ }^{48}$ Specifically, Wang et al. reported that lncRNA PTCSC3/miR-574-5p modulates cell proliferation and migration in PTC via Wnt/ $\beta$-catenin signaling. ${ }^{49}$ Therefore, the possibility of an interaction between the $\mathrm{Wnt} / \beta$-catenin signaling pathway and HOXA-AS2 in PTC was investigated in the present study. Here, the effect of the HOXA-AS2/miR-15a-5p/HOXA3 axis on the core Wnt/ $\beta$-catenin signaling factors $\beta$-catenin, c-Myc, and cyclin D1 ${ }^{50,51}$ was measured. Importantly, HOXA-AS2, miR-15a-5p, and HOXA3 significantly regulated the levels of all three proteins, suggesting HOXA-AS2/miR-15a-5p/HOXA3 axis modulation of PTC progression via the $\mathrm{Wnt} / \beta$-catenin signaling pathway.

In conclusion, the present study revealed that HOXA-AS2 functions as an oncogene in PTC and promotes progression of this disease by acting as a 
ceRNA, sponging miR-15a-5p, and regulating HOXA3. In turn, the HOXA-AS2/miR-15a-5p/HOXA3 axis modulates core proteins involved in $\mathrm{Wnt} / \beta$ catenin signaling, supporting tumor growth. These findings will significantly contribute to further research and the development of more efficient treatments for thyroid cancer in the future.

\section{ACKNOWLEDGMENTS}

The authors thank all subjects who participated in the study.

\section{AUTHOR DISCLOSURE}

There are no conflicts of interest to disclose.

\section{REFERENCES}

1. Guo C, Ma J, Deng G, et al. ZEB1 promotes oxaliplatin resistance through the induction of epithelial-mesenchymal transition in colon cancer cells. J Cancer 2017;8:3555-3566.

2. Xiong $X$, Zhu $H$, Chen $X$. Low expression of long noncoding RNA CASC2 indicates a poor prognosis and promotes tumorigenesis in thyroid carcinoma. Biomed Pharmacother 2017;93:391-397.

3. Vuong HG, Altibi AM, Abdelhamid AH, et al. The changing characteristics and molecular profiles of papillary thyroid carcinoma over time: a systematic review. Oncotarget 2017;8:10637-10649.

4. Chen W, Zheng R, Baade PD, et al. Cancer statistics in China, 2015. CA Cancer J Clin 2016;66: 115-132.

5. Wang $Y$, Wang W. Increasing incidence of thyroid cancer in Shanghai, China, 1983-2007. Asia Pac J Public Health 2015;27:NP223-229.

6. Hay ID, Lee RA, Davidge-Pitts C, et al. Long-term outcome of ultrasound-guided percutaneous ethanol ablation of selected "recurrent" neck nodal metastases in 25 patients with TNM stages III or IVA papillary thyroid carcinoma previously treated by surgery and 131I therapy. Surgery 2013;154: 1448-1454; discussion 1454-1445.

7. Nagaiah G, Hossain A, Mooney CJ, et al. Anaplastic thyroid cancer: a review of epidemiology, pathogenesis, and treatment. J Oncol 2011;2011: 542358.

8. Integrated genomic characterization of papillary thyroid carcinoma. Cell 2014;159:676-690.

9. Yang $F$, Xue $X, B i$ J, et al. Long noncoding RNA CCAT1, which could be activated by c-Myc, promotes the progression of gastric carcinoma. $J$ Cancer Res Clin Oncol 2013;139:437-445.

10. Zhang $E, H e X$, Zhang $C$, et al. A novel long noncoding RNA HOXC-AS3 mediates tumorigenesis of gastric cancer by binding to YBX1. Genome Biol 2018;19:154.

11. Wu Q, Xiang S, Ma J, et al. Long non-coding RNA CASC15 regulates gastric cancer cell proliferation, migration and epithelial mesenchymal transition by targeting CDKN1A and ZEB1. Mol Oncol 2018; 12:799-813.
12. Tsai KW, Lo YH, Liu H, et al. Linc00659, a long noncoding RNA, acts as novel oncogene in regulating cancer cell growth in colorectal cancer. Mol Cancer 2018;17:72.

13. Hu JJ, Song W, Zhang SD, et al. HBx-upregulated IncRNA UCA1 promotes cell growth and tumorigenesis by recruiting EZH2 and repressing p27Kip1/CDK2 signaling. Sci Rep 2016;6:23521

14. Li W, Zheng J, Deng J, et al. Increased levels of the long intergenic non-protein coding RNA POU3F3 promote DNA methylation in esophageal squamous cell carcinoma cells. Gastroenterology 2014;146:1714-1726.e5.

15. Li GY, Wang W, Sun JY, et al. Long non-coding RNAs AC026904.1 and UCA1: a "one-two punch" for TGF-beta-induced SNAl2 activation and epithelial-mesenchymal transition in breast cancer. Theranostics 2018;8:2846-2861.

16. Wang $H$, Liang $L$, Dong $Q$, et al. Long noncoding RNA miR503HG, a prognostic indicator, inhibits tumor metastasis by regulating the HNRNPA2B1/ NF-kappaB pathway in hepatocellular carcinoma. Theranostics 2018;8:2814-2829.

17. Li D, Chen $Y$, Mei $H$, et al. Ets-1 promoterassociated noncoding RNA regulates the NONO/ ERG/Ets-1 axis to drive gastric cancer progression. Oncogene 2018;37:4871-4886.

18. Lian Y, Li Z, Fan Y, et al. The IncRNA-HOXA-AS2/ EZH2/LSD1 oncogene complex promotes cell proliferation in pancreatic cancer. Am J Transl Res 2017:9:5496-5506.

19. Gao Y, Yu H, Liu Y, et al. Long non-coding RNA HOXA-AS2 regulates malignant glioma behaviors and vasculogenic mimicry formation via the MiR-373/ EGFR axis. Cell Physiol Biochem 2018;45:131-147.

20. Tong G, Wu X, Cheng B, et al. Knockdown of HOXAAS2 suppresses proliferation and induces apoptosis in colorectal cancer. Am J TransI Res 2017:9:4545-4552.

21. Ding J, Xie M, Lian $Y$, et al. Long noncoding RNA HOXA-AS2 represses P21 and KLF2 expression transcription by binding with EZH2, LSD1 in colorectal cancer. Oncogenesis 2017;6:e288.

22. Hua $Y$, Duan $S$, Murmann $A E$, et al. miRConnect: identifying effector genes of miRNAs and miRNA families in cancer cells. PLoS One 2011;6:e26521.
23. Fan $\mathrm{H}$, Lv P, Mu T, et al. LncRNA n335586/miR924/CKMT1A axis contributes to cell migration and invasion in hepatocellular carcinoma cells. Cancer Lett 2018;429:89-99.

24. Wang $Y$, Zeng $X$, Wang $N$, et al. Long noncoding RNA DANCR, working as a competitive endogenous RNA, promotes ROCK1-mediated proliferation and metastasis via decoying of miR-335-5p and miR-1972 in osteosarcoma. Mol Cancer 2018;17:89.

25. Xu Y, Zhang G, Zou C, et al. LncRNA MT1JP suppresses gastric cancer cell proliferation and migration through MT1JP/MiR-214-3p/RUNX3 axis. Cell Physiol Biochem 2018;46:2445-2459.

26. Zhang G, Li S, Lu J, et al. LncRNA MT1JP functions as a ceRNA in regulating FBXW7 through competitively binding to miR-92a-3p in gastric cancer. Mol Cancer 2018;17:87.

27. Wang $Y$, Sun $L$, Wang $L$, et al. Long non-coding RNA DSCR8 acts as a molecular sponge for miR-485-5p to activate $\mathrm{Wnt} / \beta$-catenin signal pathway in hepatocellular carcinoma. Cell Death Dis 2018;9:851.

28. Yang XZ, Cheng TT, He OJ, et al. LINC01133 as ceRNA inhibits gastric cancer progression by sponging miR-106a-3p to regulate APC expression and the $\mathrm{Wnt} / \beta$-catenin pathway. Mol Cancer 2018;17:126.

29. Liang $H, Y u T$, Han $Y$, et al. LncRNA PTAR promotes EMT and invasion-metastasis in serous ovarian cancer by competitively binding miR-101 $3 p$ to regulate ZEB1 expression. Mol Cancer 2018 17:119.

30. Li Y, Zeng C, Hu J, et al. Long non-coding RNASNHG7 acts as a target of miR-34a to increase GALNT7 level and regulate PI3K/Akt/mTOR pathway in colorectal cancer progression. J Hemato Oncol 2018:11:89.

31. Zhu J, Shi $H$, Liu $H$, et al. Long non-coding RNA TUG1 promotes cervical cancer progression by regulating the miR-138-5p-SIRT1 axis. Oncotarget 2017;8:65253-65264.

32. Chen X, Chen Z, Yu S, et al. Long noncoding RNA LINC01234 functions as a competing endogenous RNA to regulate CBFB expression by sponging miR-204-5p in gastric cancer. Clin Cancer Res 2018;24:2002-2014. 
33. Huang $X$, Xie $X$, Liu $P$, et al. Adam12 and Inc015192 act as ceRNAs in breast cancer by regulating miR-34a. Oncogene 2018;37:63166326.

34. Yan X, Zhang D, Wu W, et al. Mesenchymal stem cells promote hepatocarcinogenesis via IncRNAMUF interaction with ANXA2 and miR-34a. Cancer Res 2017;77:6704-6716.

35. Zhang D, Liu X. Plasma IncRNA GAS8-AS1 as a potential biomarker of papillary thyroid carcinoma in Chinese patients. 2017;2017:2645904.

36. Voutilainen PE, Multanen MM, Leppaniemi AK, et al. Prognosis after lymph node recurrence in papillary thyroid carcinoma depends on age. Thyroid 2001;11:953-957.

37. Vriens MR, Suh I, Moses W, et al. Clinical features and genetic predisposition to hereditary nonmedullary thyroid cancer. Thyroid 2009;19: 1343-1349.

38. Hombach S, Kretz M. Non-coding RNAs: classification, biology and functioning. Adv Exp Med Biol 2016;937:3-17.

39. Cao WJ, Wu HL, He BS, et al. Analysis of long non-coding RNA expression profiles in gastric cancer. World J Gastroenterol 2013;19:36583664.
40. Han $Y$, Liu $Y$, Gui $Y$, et al. Long intergenic noncoding RNA TUG1 is overexpressed in urothelial carcinoma of the bladder. J Surg Oncol 2013;107: 555-559.

41. Zhang 0 , Geng PL, Yin P, et al. Down-regulation of long non-coding RNA TUG1 inhibits osteosarcoma cell proliferation and promotes apoptosis. Asian Pac J Cancer Prev 2013;14:2311-2315.

42. Tang J, Li Y, Sang Y, et al. LncRNA PVT1 regulates triple-negative breast cancer through KLF5/betacatenin signaling. Oncogene 2018;37:4723-4734.

43. Ding D, Li C, Zhao T, et al. LncRNA H19/miR-29b3p/PGRN axis promoted epithelial-mesenchymal transition of colorectal cancer cells by acting on Wnt signaling. Mol Cells 2018;41:423-435.

44. Wang F, Yang H, Deng Z, et al. HOX antisense lincRNA HOXA-AS2 promotes tumorigenesis of hepatocellular carcinoma. Cell Physiol Biochem 2016;40:287-296.

45. Xie CR, Wang F, Zhang S, et al. Long noncoding RNA HCAL facilitates the growth and metastasis of hepatocellular carcinoma by acting as a ceRNA of LAPTM4B. Mol Ther Nucl Acids 2017;9:440-451.

46. Chen DL, Lu YX, Zhang JX, et al. Long non-coding RNA UICLM promotes colorectal cancer liver metastasis by acting as a ceRNA for microRNA-
215 to regulate ZEB2 expression. Theranostics 2017;7:4836-4849.

47. Li Z, Jiang P, Li J, et al. Tumor-derived exosomal Inc-Sox2ot promotes EMT and stemness by acting as a ceRNA in pancreatic ductal adenocarcinoma. Oncogene 2018;37:3822-3838.

48. Du HF, Ou LP, Lv CK, et al. Expression of hepaCAM inhibits bladder cancer cell proliferation via a Wnt/beta-catenin-dependent pathway in vitro and in vivo. Cancer Biol Ther 2015;16:1502-1513.

49. Wang X, Lu X, Geng Z, et al. LncRNA PTCSC3/ miR-574-5p governs cell proliferation and migration of papillary thyroid carcinoma via Wnt/ beta-catenin signaling. J Cell Biochem 2017;118: 4745-4752.

50. He TC, Sparks AB, Rago C, et al. Identification of c-MYC as a target of the APC pathway. Science 1998:281:1509-1512.

51. Tetsu 0, McCormick F. Beta-catenin regulates expression of cyclin D1 in colon carcinoma cells. Nature 1999;398:422-426.

Received for publication May 29, 2018; accepted after revision October 27, 2018.

Published online: October 29, 2018. 